\title{
Self-trigger radio prototype array for GRAND
}

\section{Yi Zhang, ${ }^{a, *}$ Haoning He ${ }^{a}$ and Pengfei Zhang ${ }^{b}$ on behalf of the GRAND Collaboration} (a complete list of authors can be found at the end of the proceedings)

${ }^{a}$ Key Laboratory of Dark Matter and Space Astronomy, Purple Mountain Observatory, Chinese Academy of Sciences, 210023 Nanjing, Jiangsu, China

${ }^{b}$ Xidian University, 266 Xinglong Section of Xifeng Road, 710126 Xian, Shaanxi, China

E-mail: zhangyi@pmo.ac.cn

The GRANDProto300 (GP300) array is a pathfinder for the Giant Radio Array for Neutrino Detection (GRAND) project. The deployment of the array, consisting of 300 antennas, will start in 2021 in a radio-quiet area of $\sim 200 \mathrm{~km}^{2}$ near Lenghu ( 3000m a.s.l.) in China. Serving as a test bench, the GP300 array is expected to pioneer techniques of autonomous radio detection including identification and reconstruction of nearly horizontal cosmic-ray (CR) air showers. In addition, the GP300 array is at a privileged position to study the transition between Galactic and extragalactic origins of cosmic rays, due to its large effective area and the precise measurements of both energy and mass composition for CRs with energies ranging from $30 \mathrm{PeV}$ to $1 \mathrm{EeV}$. Using the GP300 array we will also investigate the potential sensitivity for radio transients such as Giant Radio Pulses and Fast Radio Bursts in the 50-200 MHz range.

$37^{\text {th }}$ International Cosmic Ray Conference (ICRC 2021)

July 12 th - 23rd, 2021

Online - Berlin, Germany

\footnotetext{
*Presenter
} 


\section{Introduction}

The radio technique [1,2] for detecting both cosmic rays and neutrinos at energies above around $100 \mathrm{PeV}$ has matured. The Giant Radio Array for Neutrino Detection (GRAND)[3] will use a huge number of antennas to detect radio emission generated by extensive air showers (EASs) that are initiated by ultra-high-energy (UHE) particles in the atmosphere. GRAND will consist of roughly 20 separate, independent sub-arrays of approximately 10000 radio antennas each, totaling a combined area of $200000 \mathrm{~km}^{2}$. GRAND is proposed as a large area observatory with unprecedented sensitivity for observations of UHE neutrinos, cosmic rays and gamma rays. It is expected to give revolutionary insights into the origin of UHE Cosmic Rays (CR) and the nature of UHE neutrino sources.

The GRANDProto300 (GP300) experiment is the 300-antenna pathfinder stage of GRAND. There are multiple goals for this stage. First of all, GP300 will validate GRAND as a standalone radio detection array and optimize the self-trigger techniques. Working as a self-triggering system can fully exploit the potential of the radio-detection technique. This is a challenge, as the background sources (high voltage power lines or transformers, planes, thunderstorms, etc.) dominate transient radio signals in the tens-of- $\mathrm{MHz}$ frequency range. Nevertheless, the principle for such a selftriggering mode for an EAS radio detector has been validated on a small-scale prototype array $[4,5]$. In GP300, we will develop the algorithms to reject background and identify EAS events with improved efficiency.

Secondly, neutrino-induced EASs, which are of the major interest to GRAND, are nearhorizontal, with zenith angles above $\sim 85^{\circ}$. GP300 will be an ideal test bench to improve the reconstruction algorithm for the direction, energy and air shower development, and evaluate the quality of the reconstruction procedure.

Finally, as the GP300 array will cover $200 \mathrm{~km}^{2}$ and from its data we will be able to precisely measure the energy and mass composition for CRs from $30 \mathrm{PeV}$ to $1 \mathrm{EeV}$, and to investigate the $\mathrm{CR}$ energy spectrum and anisotropy at the transition between Galactic and extragalactic origins of cosmic rays. The GP300 array will potentially also be sensitive to radio transients such as Giant Radio Pulses and Fast Radio Bursts at 50-200 MHz range.

\section{The GP300 detector}

The GP300 array, consisting of 300 detection units, covers a $200 \mathrm{~km}^{2}$ radio-quiet area in the western part of China.

\subsection{Deployment site and layout}

The radio background noise level is an important factor for the autonomous radio detection for air showers. In our site-selection, we set the requirement that the integrated power of the stationary noise level would be less than twice the irreducible level due to Galactic and thermal ground emission in the 50-200 MHz. The other considerations are that there are no transient radio emission sources, around such as high-voltage power lines, windmills, etc.

We have started the site survey in August 2017, and several candidate sites have been visited in the Chinese provinces of Qinghai, Gansu, Xinjiang, Yunnan, and Inner-Mongolia. For two of 
these sites we are now evaluating the ease of access, infrastructure, support by local authorities, and possible extension to the GRAND10k stage.

One candidate site close to the town of Lenghu in Qinghai Province was selected in July 2019. The long-term measurement facilities were deployed in the summer of 2019, to measure the time dependent behavior of the radio environment. Another candidate is in a natural preservation zone near Dunhuang in Gansu Province.

A data collection center is built at the site, as shown in Fig 1. The first 100 antennas of GP300 have been manufactured and tested, and will be installed at the site in summer 2021. The installation of the other 200 antennas is scheduled to be started in the middle of 2022.

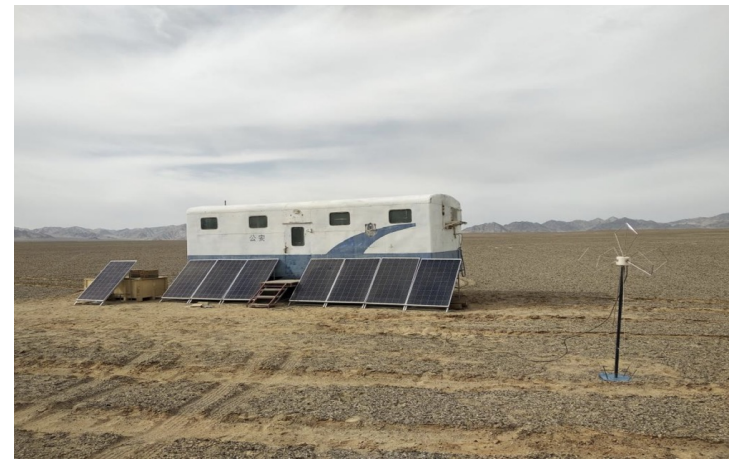

Figure 1: A picture of the data collection center at the site.

The layout of GP300 covers an area of $200 \mathrm{~km}^{2}$ with a regular grid using a $1 \mathrm{~km}$ antenna spacing, combined with a denser infill of 85 antennas with $500 \mathrm{~m}$ spacing and a core of 27 detectors on a $250 \mathrm{~m}$ grid.

\subsection{Antennas}

GRAND will detect radio signals from air showers initiated by Earth-skimming neutrinos, which arrive with zenith angles close to $90^{\circ}$. The GP300 antenna is designed to achieve a high detection efficiency for close to horizontal showers, therefore it is dubbed as the Horizontal Antenna. The height of the antennas is set to $3 \mathrm{~m}$ above ground to decrease the diffraction effect of radio waves off the ground.

Fig. 2 shows the schematic view of an antenna unit of GP300. The antenna unit is composed of 5 radiation arms. Two pairs of arms are symmetrical in the east-west and north-south directions, forming two horizontal linear polarization dipoles. The single arm in the vertical direction constitutes a monopole. The detector is operated at frequencies in the range of 50-200 MHz. The upper frequency will allow the detection of the radio Cherenkov-cone compared to previous experiments [4, 6-8]. As GP300 has a larger frequency range, a higher signal-to-noise ratio (SNR) and better reconstruction are expected [9].

\subsection{DAQ}

The analog signal in each antenna is first amplified by a $22 \mathrm{~dB}$ low noise amplifier (LNA), and then sent over a $5 \mathrm{~m}$ cable to the main board where the signal is filtered in the $50-200 \mathrm{MHz}$ 


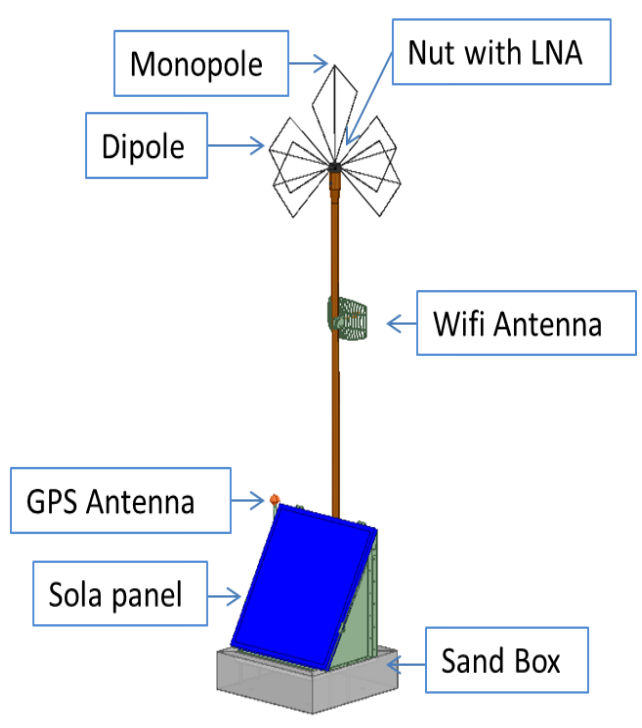

(a)

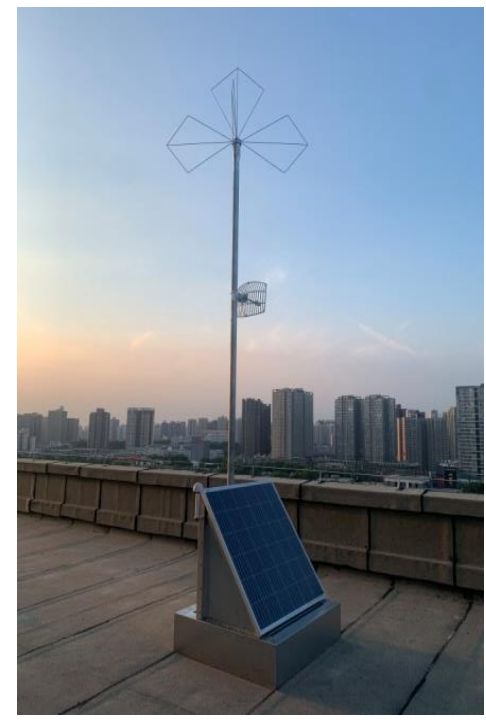

(b)

Figure 2: (a) Schematic view of the GP300 antenna unit. (b) Picture of a prototype.

frequency band and optionally amplified. Afterwards it is digitized using a 14-bit ADC (AD9694) running at a sampling rate of 500 Megasamples/s.

The digitized data is processed using a Zync FPGA with hardcore CPU (Xilinx XCZU5CG). Adjustable digital notch filters are used to reject continuous-wave emitters that may appear in this band. The FPGA will also process a real-time Fourier transform (FFT) of the data that can be used for the monitoring of radio background and searching for transient astronomical phenomena, such as FRBs.

We will transfer data to the central data acquisition (DAQ) room via WiFi technology, which allows for a throughput of $38 \mathrm{MB} \mathrm{s}^{-1}$ per sector of about 50 antennas, sufficient for our needs.

\subsection{Trigger}

We have designed three consecutive levels to progressively reduce the background:

( T0 ) A zeroth-level trigger (T0) is generated for one antenna channel when the amplitude of a radio signal after filtering exceeds a threshold. The threshold is set at $5 \sigma$, where $\sigma$ is the mean stationary noise at the antenna output.

( T1 ) The first-level trigger (T1) performs a pulse shape analysis. It evaluates the duration and structure of the time traces, and the signal polarization. The algorithm based on pulse duration allows to reject $95 \%$ of the background events. T1 time-stamps are sent to the central DAQ for evaluation.

( T2 ) The second-level trigger (T2) acts on the time-stamps sent by the T1 triggers and searches for time coincidences among a minimum of five detection neighboring antennas. If such a 
detection is found, a $3 \mu$ s-long time-trace from the T2-triggered detection units are transferred to the central DAQ.

In addition, there will be another mode to search for FRB-like transient radio waves. Each antenna will continuously calculate the FFT over 4096 samples in the $100-200 \mathrm{MHz}$ frequency range (i.e. $25 \mathrm{kHz}$ frequency resolution) and will sum them over periods of $10 \mathrm{~ms}$.

\subsection{Particle detector}

A particle detector array with an independent trigger is planned in the GP300 phase. The particle detector array can both validate the horizontal reconstruction of radio signals, and have complementary measurements that enrich the physics of GP300. Either Water-Cherenkov detectors or scintillator detectors can be used in GP300, however the exact design and layout of the array requires a dedicated study. Even though these arrays are triggered independently, both particle detector array and radio array will be synchronized through GPS, so that their information can be combined to obtain the electromagnetic and muon components of air showers. In addition, we are investigating the option of adding FAST fluorescence telescopes [10] to the GP300 site.

\section{Detector performances}

GP300 will be twice as large as the current phase of Auger Engineering Radio Array [6] . The denser infill will improve the statistics of CRs at the energy down to about $30 \mathrm{PeV}$, while the large scale of the array, $200 \mathrm{~km}^{2}$, makes it possible to derive sufficient statistics at the high energy end around $1 \mathrm{EeV}$. The different spacing between antennas allow to test the dependence of the array performance on the density of detection units. GP300 can detect about $10^{5}$ cosmic ray events in the energy range of $30 \mathrm{PeV}-1 \mathrm{EeV}$ after one-year operation, with an angular resolution better than $0.2^{\circ}$.

\section{Science cases}

\subsection{Air shower physics}

GP300 detector adopts the hybrid detection strategy to detect cosmic rays, by measuring the electromagnetic component via the radio array[ $[7,11]$ and the muon component via the ground array of particle detectors. From the measurement of the electromagnetic component, one can infer the depth of shower maximum and the energy of the primary cosmic ray[12], which is not sensitive to hadronic interaction models. The muon component together with the depth of shower maximum is correlated with the mass of the primary cosmic ray[13]. Therefore, with the measurements on electromagnetic component and muon component, we can test air-shower development for different hadronic interaction models.

\subsection{Galactic/extra-galactic transition}

GP300 is able to detect cosmic rays with energies beyond $30 \mathrm{PeV}$. It can detect about 100,000 cosmic ray events in the energy range of $30 \mathrm{PeV}-1 \mathrm{EeV}$ after one-year operation, and measure the energy and mass composition of cosmic rays more precisely[14]. Hence, GP300 is able to measure spectra of cosmic rays for each composition above the knee. It is widely believed that cosmic 
rays below the knee originate mostly from Galactic sources, and a transition between Galactic and extra-galactic sources is expected in the energy range between $10^{8}-10^{9} \mathrm{GeV}$ [15]. Therefore, GP300's measurement of the spectra of cosmic rays for different compositions will help us to study the cause of the formation of the knee and the Galactic/extra-galactic transition, and furthermore, to constrain the astrophysical source models of cosmic rays.

\subsection{Large scale anisotropy}

GP300 is able to constrain the existence of a large scale Northern-Hemisphere anisotropy with an amplitude of $10^{-5}$ at the lower energy end[14], due to its large event statistics. The observation on the large scale of anisotropy will help us to constrain the existence of nearby UHECR sources. Besides the measurements of spectra and mass composition as mentioned in Section 4.2, the measurement of the large scale of anisotropy will give us one more tool to constrain the contributions from extra-galactic sources and Galactic sources, and then finally help us to study the Galactic/extra-galactic transition.

\subsection{Ultra-high-energy gamma rays}

Thanks to the particle detectors, the efficiency of distinguishing UHE gamma-rays from cosmic rays, for zenith angles between $65^{\circ}$ and $85^{\circ}$ above an energy of $10^{9} \mathrm{GeV}$, is close to $100 \%$. If no gamma ray events are identified among a sample of $10^{5}$ showers detected in 2 years, the fraction of gamma ray-initiated showers will be limited to be $0.03 \%$ at the $95 \%$ C.L., while the current best limit is about $0.14 \%$ at the energy of $10^{9} \mathrm{GeV}[16,17]$. The upper limit of UHE gamma-rays flux measured by GP300 would constrain the super heavy dark matter models [16]. If GP300 is triggered by a UHE gamma-ray-initiated shower, it would allow follow-up partner experiments in the multimessenger network to search for counterparts associated with the triggered UHE gamma-ray.

\subsection{Radio Astronomy}

The GP300 sensitivity reaches $750 \mathrm{Jy}$ in the band of 100-200 MHz, making Giant Pulses from the Crab [18] detectable. The large field of view and high duty-cycle allow GP300 to monitor the full sky for Fast Radio Burst(FRBs), Giant Pulses, searching for possible counterparts for Gravitational Wave Signals, and measuring of the 21-cm signature from the epoch of reionization (EoR).

\section{Acknowledgements}

This work is supported in China by the National Key R\&D program of China under the grants 2018YFA0404202 and by the National Natural Science Foundation of China under the grants 11775233.

\section{References}

[1] F. G. Schröder, Progress in Particle and Nuclear Physics 93, 1 (2017), ISSN 0146-6410, URL https://www. sciencedirect.com/science/article/pii/S0146641016300758. 
[2] T. Huege and D. Besson, Progress of Theoretical and Experimental Physics 2017 (2017), ISSN 2050-3911, URL http://academic.oup.com/ptep/article/doi/10. 1093/ptep/ptx009/4665682.

[3] J. Álvarez Muñiz, R. Alves Batista, A. Balagopal V., J. Bolmont, M. Bustamante, W. Carvalho, D. Charrier, I. Cognard, V. Decoene, P. B. Denton, et al., Science China Physics, Mechanics \& Astronomy 63, 219501 (2019), ISSN 1869-1927, URL https://doi.org/10.1007/ s11433-018-9385-7.

[4] D. Ardouin, C. Cârloganu, D. Charrier, Q. Gou, H. Hu, L. Kai, P. Lautridou, O. Martineau-Huynh, V. Niess, O. Ravel, et al., Astroparticle Physics 34, 717 (2011), ISSN 09276505, number: 9, URL https: //linkinghub . elsevier . com/retrieve/pii/ S0927650511000041.

[5] D. Charrier, K. de Vries, Q. Gou, J. Gu, H. Hu, Y. Huang, S. Le Coz, O. Martineau-Huynh, V. Niess, T. Saugrin, et al., Astroparticle Physics 110, 15 (2019), ISSN 0927-6505, URL https://www. sciencedirect.com/science/article/pii/S0927650518302767.

[6] T. Huege (Pierre Auger), Nucl. Instrum. Meth. A 617, 484 (2010), 0906 . 4970.

[7] P. Schellart et al., Astron. Astrophys. 560, A98 (2013), 1311. 1399.

[8] P. Bezyazeekov, N. Budnev, O. Gress, A. Haungs, R. Hiller, T. Huege, Y. Kazarina, M. Kleifges, E. Konstantinov, E. Korosteleva, et al., Nuclear Instruments and Methods in Physics Research Section A: Accelerators, Spectrometers, Detectors and Associated Equipment 802, 89 (2015), ISSN 0168-9002, URL https://www. sciencedirect.com/science/article/ $\mathrm{pii/S0168900215010256.}$

[9] A. Balagopal V., A. Haungs, T. Huege, M. Renschler, F. G. Schröder, and A. Zilles, PoS ICRC2019, 184 (2020).

[10] M. Malacari, J. Farmer, T. Fujii, J. Albury, J. Bellido, L. Chytka, P. Hamal, P. Horvath, M. Hrabovský, D. Mandat, et al., Astroparticle Physics 119, 102430 (2020), ISSN 0927-6505, URL https://www.sciencedirect.com/science/article/pii/ S0927650520300037.

[11] T. Huege and Pierre Auger Collaboration, in European Physical Journal Web of Conferences (2019), vol. 210 of European Physical Journal Web of Conferences, p. 05011, 1905.04986.

[12] A. Aab, P. Abreu, M. Aglietta, E. J. Ahn, I. Al Samarai, I. F. M. Albuquerque, I. Allekotte, P. Allison, A. Almela, J. Alvarez Castillo, et al., 116, 241101 (2016), 1605.02564.

[13] The Pierre Auger Collaboration, A. Aab, P. Abreu, M. Aglietta, E. J. Ahn, I. A. Samarai, I. F. M. Albuquerque, I. Allekotte, P. Allison, A. Almela, et al., arXiv e-prints arXiv:1604.03637 (2016), 1604.03637.

[14] J. Álvarez-Muñiz, R. Alves Batista, A. Balagopal V., J. Bolmont, M. Bustamante, W. Carvalho, D. Charrier, I. Cognard, V. Decoene, P. B. Denton, et al., Science China Physics, Mechanics, and Astronomy 63, 219501 (2020), 1810.09994. 
[15] B. R. Dawson, M. Fukushima, and P. Sokolsky, Progress of Theoretical and Experimental Physics 2017 (2017), 1703.07897.

[16] A. Aab, P. Abreu, M. Aglietta, I. A. Samarai, I. F. M. Albuquerque, I. Allekotte, A. Almela, J. Alvarez Castillo, J. Alvarez-Muñiz, G. A. Anastasi, et al., 2017, 009 (2017), 1612 . 01517.

[17] A. Aab, P. Abreu, M. Aglietta, I. Al Samarai, I. F. M. Albuquerque, I. Allekotte, A. Almela, J. Alvarez Castillo, J. Alvarez-Muñiz, G. A. Anastasi, et al., 2020, E02 (2020).

[18] J. M. Cordes, N. D. R. Bhat, T. H. Hankins, M. A. McLaughlin, and J. Kern, 612, 375 (2004), astro-ph/0304495. 


\section{Full Authors List: GRAND Collaboration}

Jaime Álvarez-Muñiz ${ }^{1}$, Rafael Alves Batista ${ }^{2}$, Aurélien Benoit-Lévy ${ }^{3}$, Julien Bolmont ${ }^{4}$, Henk Brans $^{2}$, Mauricio Bustamante ${ }^{5}$, Didier Charrier ${ }^{6}$, LingMei Cheng ${ }^{7}$, Simon Chiche $^{8}$, Zigao Dai ${ }^{9}$, Rogerio M. de Almeida ${ }^{11}$, Valentin Decoene ${ }^{12}$, Peter B. Denton ${ }^{13}$, Beatriz de Errico ${ }^{14}$, Sijbrand De Jong ${ }^{2,15}$, João R. T. de Mello Neto ${ }^{14}$, Krijn D. De Vries ${ }^{16}$, Kaikai Duan ${ }^{21}$, Ran Duan ${ }^{7}$, Ralph Engel $^{17,18}$, Yizhong Fan ${ }^{21}$, Ke Fang ${ }^{22}$, QuanBu Gou ${ }^{23}$, Junhua Gu ${ }^{7}$, Claire Guépin ${ }^{19,20}$, Jianhua $\mathrm{Guo}^{21}$, Yiqing Guo ${ }^{23}$, Rene Habraken ${ }^{2,15}$, Andreas Haungs ${ }^{17}$, Haoning $\mathrm{He}^{21}$, Eric Hivon ${ }^{8}$, Hongbo $\mathrm{Hu}^{23}$, Xiaoyuan Huang ${ }^{21}$, Yan Huang ${ }^{7}$, Tim Huege ${ }^{17,10}$, Marcelo Ismerio Oliveira ${ }^{14}$, Ramesh Koirala ${ }^{25,26}$, Kumiko Kotera ${ }^{8,27}$, Wen Jiang ${ }^{24}$, Bruno L. Lago ${ }^{28}$, Sandra Le Coz ${ }^{4}$, JeanPhilippe Lenain ${ }^{4}$, Bo Liu ${ }^{24}$, Cheng Liu ${ }^{23}$, Ruoyu Liu ${ }^{25,26}$, Wei Liu ${ }^{23}$, Pengxiong Ma ${ }^{21}$, Olivier Martineau-Huynh $^{4,7,8}$, Miguel Mostafá ${ }^{29,12}$, Fabrice Mottez ${ }^{30}$, Jean Mouette ${ }^{8}$, Kohta Murase ${ }^{29,12}$, Valentin Niess ${ }^{31}$, Foteini Oikonomou ${ }^{32}$, Ziwei Ou ${ }^{33}$, Tanguy Pierog ${ }^{17}$, Lech Wiktor Piotrowski ${ }^{34}$, Simon Prunet ${ }^{35}$, Xiangli Qian ${ }^{36}$, Inge van Rens ${ }^{2}$, Valentina Richard Romei ${ }^{8}$, Markus Roth ${ }^{17}$, Fabian Schüssler $^{37}$, Dániel Szálas-Motesiczky ${ }^{2}$, Jikke Tacken ${ }^{2}$, Anne Timmermans ${ }^{2,16}$, Charles Timmermans ${ }^{2,15}$, Matías Tueros ${ }^{38,8}$, Rongjuan Wang ${ }^{24}$, Shen Wang ${ }^{21}$, Xiangyu Wang ${ }^{25,26}$, Xu $\mathrm{Wang}^{39}$, Clara Watanabe ${ }^{14}$, Daming $\mathrm{Wei}^{21}$, Feng $\mathrm{Wei}^{24}$, Thei Wijnen ${ }^{2}$, Xiangping $\mathrm{Wu}^{7,40}$, Xuefeng $\mathrm{Wu}^{41}$, Xin $\mathrm{Xu}^{24}$, Xing $\mathrm{Xu}^{21}$, Lili Yang ${ }^{33}$, Xuan Yang ${ }^{21}$, Qiang Yuan ${ }^{21}$, Philippe Zarka ${ }^{42}$, Houdun Zeng ${ }^{21}$, Bing Theodore Zhang ${ }^{12}$, Chao Zhang ${ }^{17,43,44}$, Jianli Zhang ${ }^{7}$, Kewen Zhang ${ }^{4}$, Pengfei Zhang ${ }^{24}$, Songbo Zhang ${ }^{41}$, Yi Zhang ${ }^{21}$, Hao Zhou ${ }^{45}$

${ }^{1}$ Departamento de Física de Partículas \& Instituto Galego de Física de Altas Enerxías, Universidad de Santiago de Compostela, 15782 Santiago de Compostela, Spain

${ }^{2}$ Institute for Mathematics, Astrophysics and Particle Physics (IMAPP), Radboud Universiteit, Nijmegen, Netherlands

${ }^{3}$ Université Paris-Saclay, CEA, List, F-91120, Palaiseau, France

${ }^{4}$ Sorbonne Université, Université Paris Diderot, Sorbonne Paris Cité, CNRS, Laboratoire de Physique Nucléaire et de Hautes Energies (LPNHE), 4 place Jussieu, F-75252, Paris Cedex 5, France

${ }^{5}$ Niels Bohr International Academy, Niels Bohr Institute, 2100 Copenhagen, Denmark

${ }^{6}$ SUBATECH, Institut Mines-Telecom Atlantique - CNRS/IN2P3 - Université de Nantes, Nantes, France

${ }^{7}$ National Astronomical Observatories, Chinese Academy of Sciences, Beijing 100101, China

${ }^{8}$ Sorbonne Université, CNRS, UMR 7095, Institut d'Astrophysique de Paris, 98 bis bd Arago, 75014 Paris, France

${ }^{9}$ University of Science and Technology of China, 230026 Hefei, Anhui, China

${ }^{10}$ Astrophysical Institute, Vrije Universiteit Brussel, Pleinlaan 2, 1050 Brussel, Belgium

${ }^{11}$ Universidade Federal Fluminense, EEIMVR, Volta Redonda, RJ, Brazil

${ }^{12}$ Department of Physics, Department of Astronomy \& Astrophysics, Pennsylvania State University, University Park, PA 16802, USA

${ }^{13}$ High Energy Theory Group, Physics Department,Brookhaven National Laboratory, Upton, NY 11973, USA

${ }^{14}$ Universidade Federal do Rio de Janeiro (UFRJ), Instituto de Física, Brazil

${ }^{15}$ Nationaal Instituut voor Kernfysica en Hoge Energie Fysica (Nikhef), Netherlands

${ }^{16}$ IIHE/ELEM, Vrije Universiteit Brussel, Pleinlaan 2, 1050 Brussels, Belgium

${ }^{17}$ Institute for Astroparticle Physics, Karlsruhe Institute of Technology (KIT), D-76021 Karlsruhe, Germany

18 Institute of Experimental Particle Physics (ETP), Karlsruhe Institute of Technology (KIT), D-76021

Karlsruhe, Germany

${ }^{19}$ Department of Astronomy, University of Maryland, College Park, MD 20742-2421, USA

${ }^{20}$ Joint Space-Science Institute, College Park, MD 20742-2421, USA 
${ }^{21}$ Key Laboratory of Dark Matter and Space Astronomy, Purple Mountain Observatory, Chinese Academy of Sciences, 210023 Nanjing, Jiangsu, China

${ }^{22}$ Wisconsin IceCube Particle Astrophysics Center (WIPAC) and Dept. of Physics, University of WisconsinMadison, Madison, WI 53703, USA

${ }^{23}$ Institute of High Energy Physics, Chinese Academy of Sciences, 19B YuquanLu, Beijing 100049, China

${ }^{24}$ Key Laboratory of Antennas and Microwave Technology, Xidian University, Xi' an 710071, China

25 School of Astronomy and Space Science, Xianlin Road 163, Nanjing University, Nanjing 210023, China

${ }^{26}$ Key laboratory of Modern Astronomy and Astrophysics (Nanjing University), Ministry of Education, Nanjing 210023, People's Republic of China

27 Vrije Universiteit Brussel (VUB), Dienst ELEM, Pleinlaan 2, B-1050, Brussels, Belgium

${ }^{28}$ Centro Federal de Educação Tecnológica Celso Suckow da Fonseca, Nova Friburgo, Brazil

${ }^{29}$ Center for Multimessenger Astrophysics, Pennsylvania State University, University Park, PA 16802, USA

${ }^{30}$ LUTH, Obs. de Paris, CNRS, Université Paris Diderot, PSL Research University, 5 place Jules Janssen, 92190 Meudon, France

${ }^{31}$ Université Clermont Auvergne, CNRS/IN2P3, LPC, F-63000 Clermont-Ferrand, France.

32 Institutt for fysikk, NTNU, Trondheim, Norway

33 School of Physics and Astronomy, Sun Yat-sen University, Zhuhai 519082, China

${ }^{34}$ Faculty of Physics, University of Warsaw, Pasteura 5, 02-093 Warsaw, Poland

${ }^{35}$ Laboratoire Lagrange, Université Côte d'Azur, Observatoire de la Côte d'Azur, CNRS, Parc Valrose, 06104 Nice Cedex 2, France

${ }^{36}$ Department of Mechanical and Electrical Engineering, Shandong Management University, Jinan 250357, China

${ }^{37}$ IRFU, CEA, Université Paris-Saclay, F-91191 Gif-sur-Yvette, France

38 Instituto de Física La Plata, CONICET, Boulevard 120 y 63 (1900), La Plata, Argentina

${ }^{39}$ Department of Mechanical and Electrical Engineering, Shandong Management University, Jinan 250357,China.

${ }^{40}$ Shanghai Astronomical Observatory, Chinese Academy of Sciences, 80 Nandan Road, Shanghai 200030, China

41 Purple Mountain Observatory, Chinese Academy of Sciences, Nanjing 210023, China

${ }^{42}$ LESIA, Observatoire de Paris, CNRS, PSL/SU/UPD/SPC, Place J. Janssen, 92195 Meudon, France

${ }^{43}$ Kavli Institute for Astronomy and Astrophysics, Peking University, Beijing 100871, China

${ }^{44}$ Department of Astronomy, School of Physics, Peking University, Beijing 100871, China

45 Tsung-Dao Lee Institute \& School of Physics and Astronomy, Shanghai Jiao Tong University, 200240 Shanghai,China 